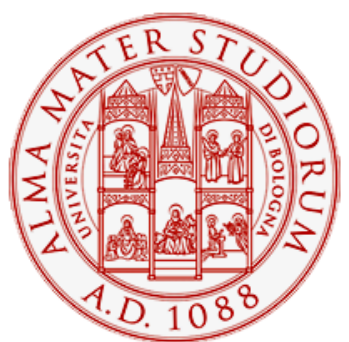

Alma Mater Studiorum - Università di Bologna DEPARTMENT OF ECONOMICS

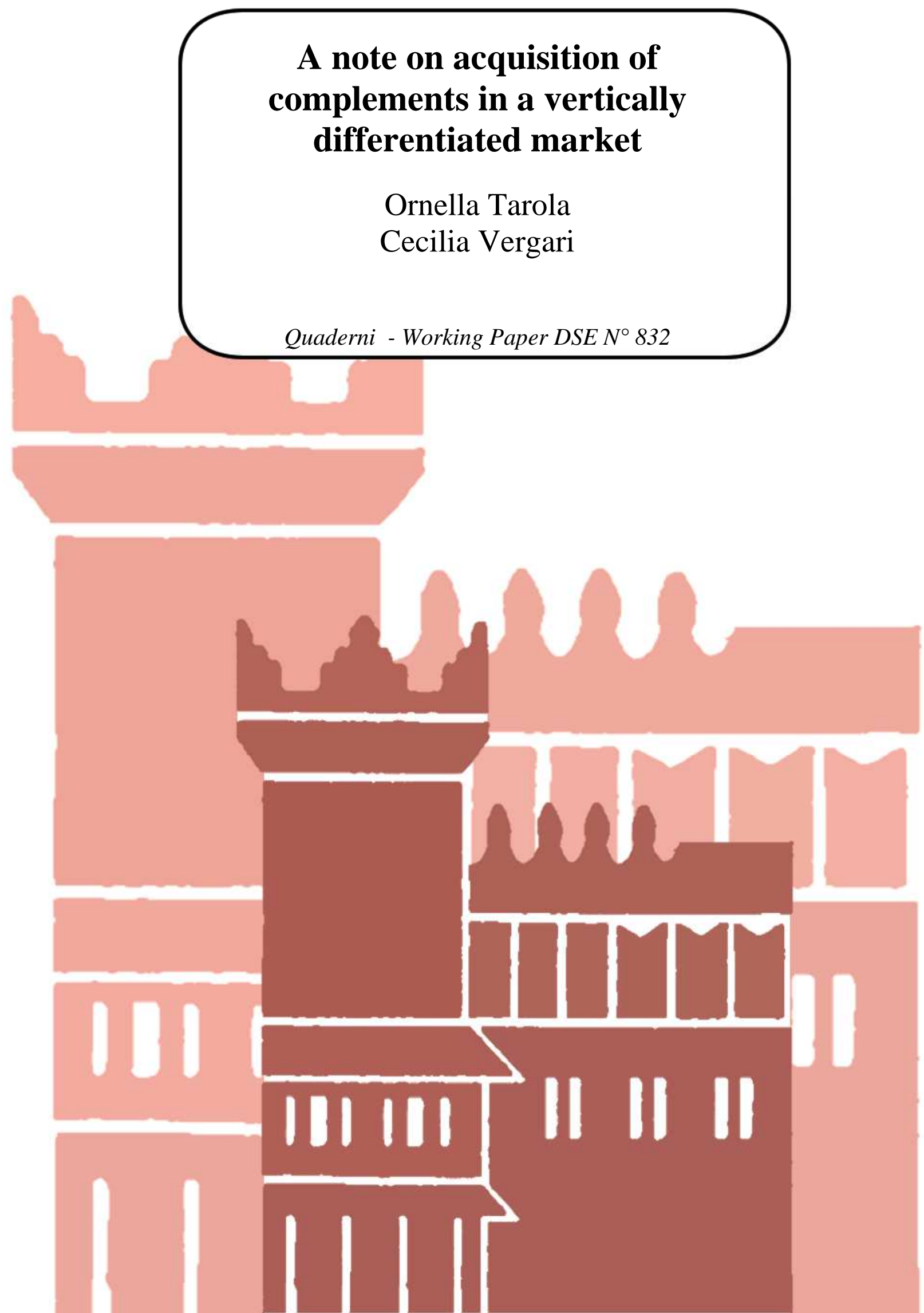




\title{
A note on acquisition of complements in a vertically differentiated market*
}

\author{
Ornella Tarola ${ }^{\dagger}$ and Cecilia Vergari ${ }^{\ddagger}$
}

\begin{abstract}
This note is concerned with the effects of joint ownership of complements when they are vertically differentiated. We provide strong arguments for the positive nature of network integration among firms, while showing at the same time that, in some circumstances, anti-competitive consequences can be observed under acquisition.
\end{abstract}

JEL classification: L1, L4.

Keywords: Bundling, Acquisition, Vertical Differentiation

*We have benefitted from useful comments from Jean J. Gabszewicz and Andrea Mantovani. Any remaining errors are ours.

†DISS, University of Rome "La Sapienza", Piazzale Aldo Moro 5, 00100 Rome, Italy, corresponding author, e-mail: ornella.tarola@uniroma1.it, phone num: $+39(0) 6-49910253$.

${ }^{\ddagger}$ Department of Economics, University of Bologna, Strada Maggiore, 45 I-40125 Bologna Italy. email: cecilia.vergari@unibo.it. 


\section{Introduction}

This note is concerned with the effects of M\&As between producers of asymmetric complementary goods in vertically differentiated markets. Since the pioneristic work by Cournot (1938), the view that an intergrated monopoly may be beneficial to consumers is generally shared. The joint ownership of complements benefits consumers as it removes a problem of double marginalization while possibly passing through to consumers further gains. ${ }^{1}$ This clear-cut merger's implication is mitigated in oligopoly markets. Indeed, while the joint ownership determines "vertical" integration between producers of different components, it also decreases competition in the market while inducing "horizontal" integration between producers of a given component. While the former effect pushes prices downwards, the latter pushes prices upward (Economides and Salop 1992). ${ }^{2}$

In this note, we re-examine the above evoked competing effects in a framework of vertical differentiation. We put the argument in the simpest possible setting. There is an incumbent monopolist that produces a base good. This good can be equipped with a complementary component, which is produced by the monopolist itself and a potential entrant. The value of the base good increases with the quality of the complementary component with which it is bundled. The monopolist produces a complementary component whose quality is assumed to be lower than that produced by the potential entrant. When facing the rival, the monopolist can either deter entry by selling pure bundles, so that only the low quality system is marketed; or accept entry by offering mixed bundles, so that both qualities are available; or acquire the entrant, in which case only the high quality system is put on sale. We prove that, if allowed, acquisition would be always observed at equilibrium. Otherwise mixed bunbling would arise. Further, we show that acquisition could be welfare improving under some conditions on market size and quality differential among complements. This holds as in vertically differentiated markets, under joint ownership, on one hand the traditional

\footnotetext{
${ }^{1}$ For example, when the quality of a two-products system is determined by the minimum of the qualities of its components, an integrated monopoly in which both complementary goods are sold by a single integrated firm dominates complementary monopolies (namely, independent ownership) in terms of welfare implications: in fact, the first entails higher quality goods' and higher market coverage (Economides 1999, Maruyama et al. 2011) while neutralizing the vertical externality of double marginalization.

${ }^{2}$ Recent contributions show that welfare losses may be also due to the practice of mixed bundling by merged firms or foreclosure (Choi 2008, Flores-Fillol and Moner-Colonques 2011). Interestingly, both these pratices develop along a reducingcompetition dimension.
} 
welfare enhancing effect (taking place when the double marginalization is removed) can be magnified by an increase in the average quality of consumption; on the other hand, the negative horizontal externality can play a minor role when the market is sufficiently large. 3 These arguments can be valuable for competition agencies when evaluating mergers in vertically differentiated markets.

\section{The basic framework}

We consider a market with a monopolist $I$ and a potential entrant $E$. The monopolist sells a base good of quality $\bar{u}$ to a population of consumers identified by the parameter $\theta \in[a, b], 0 \leq a<b$ and uniformly distributed with density equal to 1 . Both the monopolist $I$ and the potential entrant $E$ can sell a complementary asset of quality $v_{I}$ and $v_{E}$, respectively, with $v_{E}>v_{I}$. The complementary good does not bring any value to the consumers who do not buy the base good. Still, it allows the base good to perform better. Let $u_{i}$ be the overall quality of the base good when sold equipped with the complementary variant $v_{i}$, $i=I, E, u_{E}>u_{I}>\bar{u}$ holds. ${ }^{4}$ The average cost of production of both the base good and the complementary assets are assumed to be constant and, without loss of generality, equal to zero. When facing the rival, the monopolist can deter entry by selling pure bundles $(P B)$; accept entry by offering mixed bundles $(M B)$, namely both the base good without further complementary variant and the low quality bundle; acquire the entrant and thus sell on its behalf the complementary high quality variant $v_{E}$. In this latter case, the incumbent has to pay an acquisition price to the entrant while avoiding open competition.

We analyse these scenarios in turn, from both a private and a social welfare point of view.

\section{Equilibrium analysis}

Bundling strategy Let us first consider the bundling strategy. Under pure bundling, the incumbent extends the monopoly power to the complementary market and sells only a good of quality $u_{I}>\bar{u}$. Each consumer $\theta$ can either buy the low quality bundle and get utility $\theta u_{I}-P_{I}$ or not buying at all and get a nil utility. The incumbent equilibrium profit

\footnotetext{
${ }^{3}$ Quite interestingly, although this result heavily rests on the assumption that the complementary components are vertically differentiated, it is in line with the rationale stated by Economides and Salop (1992).

${ }^{4}$ As it will be clear after the equilibrium analysis, the opposite case in which the incumbent can offer a high quality bundle is trivial: at equilibrium pure bundling always arises.
} 
is $\prod_{I}^{P B}=\frac{1}{4} u_{I} b^{2}$; the equilibrium consumer surplus is $C S^{P B}=\frac{1}{8} u_{I} b^{2}{ }^{5}$

Under mixed bundling, each consumer can either buy only the base good at price $p_{u}$ and get utility $\theta \bar{u}-p_{u}$; or buy the base good equipped with the low quality (resp. high quality) complementary variant at some price $P_{I}$ (resp. $p_{u}+r_{E}$, where $r_{E}$ is the price of $v_{E}$ sold by the entrant) in which case the utility is $\theta u_{I}-P_{I}$ (resp. $\theta u_{E}-p_{u}-r_{E}$ ); or refrain from buying. At equilibrium the incumbent finds it profitable to quote for the base good a price $p_{u}$ so high that no consumer buys the base good alone. Then, the first consumer willing to buy something is $\bar{\theta}=\frac{P_{I}}{u_{I}}{ }^{6}$ Accordingly, the incumbent and the entrant profits functions write as, respectively:

$$
\begin{aligned}
& \Pi_{I}^{M B}\left(P_{I}, p_{u}, r_{E}\right)=(\breve{\theta}-\bar{\theta}) P_{I}+(b-\breve{\theta}) p_{u}, \\
& \Pi_{E}^{M B}\left(P_{I}, p_{u}, r_{E}\right)=(b-\breve{\theta}) r_{E} .
\end{aligned}
$$

where $\breve{\theta}=\frac{p_{u}+r_{E}-P_{I}}{u_{E}-u_{I}}$ is the consumer indifferent between buying the low quality bundle and the base good equipped with the high quality complementary variant. Price competition leads to the following equilibrium prices, profits and consumer surplus:

$$
\begin{aligned}
p_{u} & =\frac{1}{6} u_{I}+\frac{1}{3} u_{E}, P_{I}=\frac{1}{2} b u_{I}, r_{E}=\frac{1}{3} b\left(u_{E}-u_{I}\right) ; \\
\Pi_{I}^{M B} & =\frac{1}{36} b^{2}\left(5 u_{I}+4 u_{E}\right), \Pi_{E}^{M B}=\frac{1}{9}\left(u_{E}-u_{I}\right) b^{2} ; \\
C S^{M B} & =\frac{4 u_{E}^{2}(2 b-1)^{2}+u_{I} u_{E}(b-2)^{2}+u_{I}^{2}\left(2 b-8 b^{2}+1\right)}{72\left(u_{E}-u_{I}\right)} .
\end{aligned}
$$

Comparing these two scenarios, we find that the incentive to mixed bundling always dominates that to pure bundling, namely $\Pi_{I}^{M B}-\Pi_{I}^{P B}>$ 0 . Note that under these two scenarios the equilibrium demands for the incumbent coincide. ${ }^{7}$ However, under $M B$ the incumbent benefits from the presence of the high quality variant. Indeed, he can put in place a sort of price discrimination, thereby raising the price of the base good $p_{u}$ to such an extent that this latter is sold only to consumers buying it equipped with a variant, whatever it is. As for consumer surplus we find $C S^{M B}-C S^{P B}>0$. Mixed bundling is thus superior from the social welfare point of view, as it is preferred by the firms as well as by consumers.

\footnotetext{
${ }^{5}$ Given the demand $b-\frac{P_{I}}{u_{I}}$, it is straightforward to find these equilibrium variables. Note also that for this and the subsequent equilibria we find, we do not assume $a$ priori that the market is, or is not, covered.

${ }^{6}$ The proof of this result is available from the authors upon request. See Gabszewicz, Sonnac and Wauthy (2001) for an interesting analysis related to this point.

${ }^{7}$ At equilibrium the demand for the incumbent is $(b-\bar{\theta})=\frac{1}{2} b$ under $P B$ and $(\breve{\theta}-\bar{\theta})+(b-\breve{\theta})=\frac{1}{6} b+\frac{1}{3} b=\frac{1}{2} b$ under $M B$.
} 
Acquisition strategy Let us now move to consider the case when the incumbent acquires at some acquisition price $P_{A}$ the entrant thereby avoiding open competition. A priori, in this scenario the monopolist can sell all three variants, combinations of two of them, or only one of them, namely the top combination $u_{E}$ at price $P_{E} \cdot{ }^{8}$ Suppose that the incumbent sells all the three variants. Then, the profit maximization problem writes as

$$
\max _{p_{u}, P_{I}, P_{E}}\left((\widetilde{\theta}-\bar{\theta}) p_{u}+(\breve{\theta}-\widetilde{\theta}) P_{I}+(b-\breve{\theta}) P_{E}\right)
$$

where $P_{E}$ is the price of the high quality bundle; the indifferent consumer types are $\bar{\theta}=\frac{p_{u}}{\bar{u}}, \widetilde{\theta}=\frac{P_{I}-p_{u}}{u_{I}-\bar{u}}$ and $\breve{\theta}=\frac{P_{E}-P_{I}}{u_{E}-u_{I}}$. Equilibrium prices, profit and consumer surplus are then:

$$
\begin{aligned}
P_{E}^{A c q} & =\frac{1}{2} b u_{E}, P_{I}^{A c q}=\frac{1}{2} b u_{I}, p_{u}^{A c q}=\frac{1}{2} b \bar{u}, \\
\Pi_{I}^{A c q} & =\frac{1}{4} u_{E} b^{2} \\
C S^{A c q} & =\frac{1}{8} u_{E} b^{2} .
\end{aligned}
$$

At equilibrium the incumbent sells only the high quality variant. ${ }^{9} \mathrm{~A}$ positive market expansion effect of selling more than one quality is overcompensated by a negative cannibalization effect. ${ }^{10}$

Equilibrium strategy It remains now to set the equilibrium analysis, namely to see whether the incentive to acquisition dominates that to mixed bundling. To this aim, notice that, in order to be preferred over the alternative, the acquisition proposal should yield the entrant $E$ a gain at least equal to the profits it would get under turning off the proposal, namely $\Pi_{E}^{M B}$. Of course, it follows that the acquisition price $P_{A}$ has to be equal to $\Pi_{E}^{M B}$. Furthermore, it is convenient for the incumbent $I$ to make such a proposal if, and only if, profits obtained when acquiring the entrant after paying the acquisition price are larger than the profits it would get in the alternative scenario, namely $\Pi_{I}^{M B}$. As $\Pi_{I}^{A c q}-P_{A}>\Pi_{I}^{M B}$ with $P_{A}=\Pi_{E}^{M B}$, we can state the following.

Proposition 1 At equilibrium, acquisition always prevails over bundling.

\footnotetext{
${ }^{8}$ Selling only the low combination $u_{I}$ is the pure bundling scenario.

${ }^{9}$ In fact $P_{I}^{A c q}$ and $p_{u}^{A c q}$ are such that the demands for the base good alone and the low quality bundle are equal to zero

${ }^{10}$ Since the pioneristic contribution of Mussa and Rosen (1978), a huge amount of literature has considered the profitability of quality discrimination by a monopolist in a vertically differentiated market. See Acharyya (1998), Gabszewicz and Wauthy (2002), inter alia.
} 


\section{Acquisition and welfare}

We have shown above that the privately optimal strategy is acquisition. We next wonder whether a competition authority would allow such an acquisition, taking into account that the outside option is $M B$. For sake of simplicity, as a measure of welfare we consider consumer surplus (CS). Notice however that, moving to the social welfare as alternative measure of welfare would not affect our qualitative results.

Comparing the equilibrium consumer surplus under $M B$ vs $A c q$, we find:

$$
C S^{M B}-C S^{A c q}=\frac{\left(2 b u_{I}-u_{I}+u_{E}(b-2)\right)\left(u_{E}(7 b-2)-4 b u_{I}-u_{I}\right)}{72\left(u_{E}-u_{I}\right)} .
$$

The sign of $\left(C S^{M B}-C S^{A c q}\right)$ depends on the market size, $b$ and the quality differential $\frac{u_{E}}{u_{I}}=U$; in particular, it is the same as the sign of:

$$
((2 b-1)+U(b-2))(U(7 b-2)-(4 b+1)) .
$$

Simple calculations reveal that:

- for $b>2$ or $b<2 / 7, C S^{A c q}<C S^{M B}$ always holds;

- for $b \in(1,2), C S^{A c q}>C S^{M B} \Longleftrightarrow U>\bar{U}=\frac{(2 b-1)}{(2-b)}$;

- finally, for $b \in(2 / 7,1), C S^{A c q}>C S^{M B} \Longleftrightarrow U>\tilde{U}=\frac{(4 b+1)}{(7 b-2)}$.

Under $M B$, both qualities are on sale whereas under $A c q$, only the high quality good is marketed. So, moving from $M B$ to $A c q$ entails four effects on consumers.

A first negative effect linked to the reduction of the number of qualities, in particular some of the consumers that under $M B$ buy the low quality good, do not buy anymore under $A c q$. This negative effect is stronger, the higher is $b$, that is the heterogeneity among consumers. ${ }^{11}$ A second positive effect is due to the fact that some of the consumers that under $M B$ buy the low quality good are now willing to buy the high quality good under Acq. This positive composition effect of demand is particularly strong when the quality gap, $\frac{u_{E}}{u_{I}}$, is high. Finally, when switching from $M B$ to $A c q$, we have two contrasting effects on prices. On one hand competition becomes milder as we now have only one firm rather than two; this negative competition effect is not very strong when the market is large (it is as if each firm had its own segment of the market), in contrast it becomes strong when the market is

\footnotetext{
${ }^{11}$ Indeed, ceteris paribus, the higher $b$ the higher is the heterogeneity among consumers, namely the lower is the amount of consumers willing to buy the high quality variant rather than to stop buying.
} 
small, as competition in this case is tough. On the other hand under $A c q$ the monopolist internalizes the double marginalization problem that is at work under $M B$; this is a positive pricing effect for consumers and it is stronger the lower is $b .{ }^{12}$ Comparing equilibrium prices we find that for high (resp. low) values of $b$, namely $b>2$ (resp. $b<1$ ) the equilibrium price of the high quality variant under acquisition is higher (resp. lower) than the corresponding one under mixed bundling.

The first negative effect linked to the reduction of the number of varieties dominates when $b$ is sufficienty large, thus driving the preference for $M B$ over $A c q$. The third negative competition effect dominates when $b$ is particularly small, thus driving the preference for $M B$ over $A c q$. Finally, for intermediate values of $b$, the four effects balance in such a way that the second positive effect dominates as long as the quality gap is high, thus driving the preference for $A c q$ over $M B$.

The following Proposition summarizes our previous considerations. ${ }^{13}$

Proposition 2 As long as the market size is either particularly large or small, acquisition is welfare detrimental. In contrast, for an intermediate market size acquistion is welfare improving if and only if the quality gap is sufficiently high.

This result represents something to chew on by competition agencies when evaluating proposed mergers. Indeed, it shows that in vertically differentiated markets, the traditional welfare enhancing effect (taking place when the double marginalization is removed) can be magnified by a positive composition effect of demand. Further, as the negative horizontal externality can play a minor role when the market is sufficiently large, it may well happen that, even when enhancing a monopoly structure, M\&As can benefit consumers.

\section{Conclusion}

This note sheds light on the effects of joint ownership of complements when they are vertically differentiated. Although our model is highly stylized, still it provides strong arguments for the positive nature of network integration among firms in vertically differentated markets, while showing at the same time that, under some circumstances, anti-competitive consequences can be observed.

\footnotetext{
${ }^{12}$ These two effects on prices resemble

the horizontal and vertical externatlities observed by Economides and Salop (1992).

${ }^{13}$ The full proof Proposition 2 involves tedious comparisons and is available from the authors upon request (also with comparison of the total social welfare under MB vs Acq).
} 


\section{References}

[1] Acharyya, R., 1998. Monopoly and product quality: separating or pooling menu? Economics Letters 61, 187-194

[2] Choi, J. P., 2008. Mergers with bundling in complementary markets, Journal of Industrial Economics 56, 553-577.

[3] Economides, N. 1999. Quality choice and vertical integration, International Journal of Industrial Organization 17: 903-914.

[4] Economides, N. and Salop, S. C., 1992. Competition and integration among complements, and network market structure, Journal of Industrial Economics 40, 105-123.

[5] Flores-Fillol, R., and Moner-Colonques, R., 2011. Endogenous Mergers of Complements with Mixed Bundling, Review of Industrial Organization 39: 231-251.

[6] Gabszewicz J.J. and Wauthy, X., 2002. Quality underprovision by a monopolist when quality is not costly. Economics Letters 77 (1), 65-72.

[7] Gabszewicz, J.J., Sonnac, N. and Wauthy, X., 2001. On price competition with complementary goods, Economics Letters 70(3), 431-437.

[8] Maruyama M., Minamikawa K. and Zennyo Y., 2011 A Note on the Desirability of Merger among Complements, Journal of Industry Competition and Trade 11, 57-65

[9] Mussa, S. and Rosen, S., 1978. Monopoly and product quality, Journal of Economic Theory 18, 301-317. 


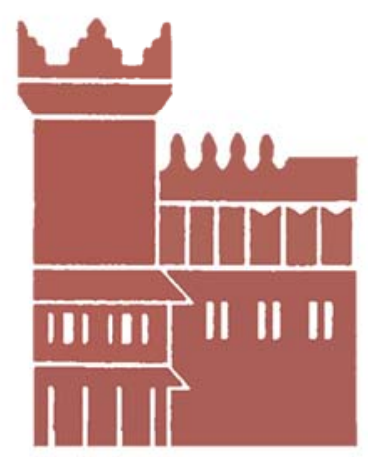

Alma Mater Studiorum - Università di Bologna DEPARTMENT OF ECONOMICS

Strada Maggiore 45

40125 Bologna - Italy

Tel. +39051 2092604

Fax +390512092664

http://www.dse.unibo.it 